\title{
The application prospects of artificial intelligence in the field of mental health and hygiene
}

\author{
$1^{\text {st } Y a n g ~ f e i ~}{ }^{1, a}, 2^{\text {nd }}$ Xie Jiashun ${ }^{2, b}, *$ Corresponding Author: Zhong zhi bing(male, professor $)^{3, \mathrm{c}}$ \\ ${ }^{1}$ Department of Applied Psychology, Jiangxi University of Traditional Chinese Medicine, Nanchang, China. \\ ${ }^{2}$ Department of Applied Psychology, Jiangxi University of Traditional Chinese Medicine, Nanchang, China. \\ ${ }^{3}$ Department of Applied Psychology, Jiangxi University of Traditional Chinese Medicine, Nanchang, China.
}

\begin{abstract}
At present, clinical psychologists at home and abroad have applied artificial intelligence to the treatment of mental illness and have achieved certain results. Artificial intelligence has tried to be applied to various fields of mental health and hygiene. This article reviews the application of artificial intelligencerelated technologies in mental health, including the application of artificial intelligence to mental illness identification and early warning intervention, and the application of artificial intelligence to the mental level of the elderly, and discusses its advantages, disadvantages and prospects.
\end{abstract}

\section{Introduction}

Artificial intelligence technology is abbreviated as a discipline. As a discipline, it is a comprehensive edge discipline developed on the basis of computer science, cybernetics, informatics, neuropsychology, philosophy, linguistics and other disciplines. However, as to the definition of artificial intelligence technology, there is no unified expression so far, and scholars of different disciplines and different disciplinary backgrounds have different understandings and understandings of artificial intelligence technology. Broadly speaking, artificial intelligence technology is about the intelligent behavior of man-made objects. This intelligent behavior generally includes perception, learning, reasoning, communication, and behavior in complex environments [1]. In addition, from the engineering point of view, artificial intelligence technology is to use artificial methods to make machines have functions related to human intelligence, such as judgment, reasoning, proof, perception, understanding, thinking, identification, planning, design, learning, and problem solving. It is the realization of human intelligence on the machine [2].

\section{The application of artificial intelligence in mental illness}

At present, artificial intelligence is often used to promote the early detection of diseases, to understand the progress of the disease, to optimize the choice of drugs and therapeutic doses, and to discover new treatment methods [3]. The biggest advantage of artificial intelligence is that it can perform rapid pattern analysis on a large number of data sets. Among them, the most successful medical fields using pattern recognition include ophthalmology, cancer detection, and imaging. In these fields, artificial intelligence may be as good as or even better than experienced clinicians in evaluating image abnormalities or subtle differences that cannot be detected by the human eye [4]. Human learning ability is limited by learning experience, knowledge sources and life experience, but artificial intelligence machines can quickly synthesize information from unlimited medical information sources, and through computer analysis, reveal the trends and associations of human behavior and patterns. It is often difficult to extract this information [4]. Although smart machines are unlikely to completely replace clinicians, smart systems are increasingly being used for clinical diagnosis and treatment [3]. The rise of artificial intelligence based on big data analysis has brought dawn to psychologists and patients. With the fourth industrial revolution initiated in the 21 st century, artificial intelligence has become a hot area of technological development today. The application of artificial intelligence in the whole course of mental illness will profoundly affect the evaluation, prediction and treatment of mental illness.

\subsection{Analysis of acoustic characteristics of mental illness based on artificial intelligence}

The fifth edition of the Diagnostic and Statistical Manual of Mental Disorders describes "psychomotor block" as "decrease in speaking volume, change in pitch, decrease in number, narrowing of speech content, or silence", suggesting that acoustic performance may be related to mental illness Related. Studies have shown that several quantitative measurements of speech acoustics are closely related to the subjective measurement of the emotional 
state and severity of symptoms in patients with major depression [6].

Gillespie [7] and others used speech analysis technology to objectively evaluate the voice of adult patients with aphasia to understand the changes in their emotional state. This type of research suggests that, based on the characteristics of speech, machine learning methods and the use of speech variables to establish an effective automatic recognition model of mental illness may provide objective indicators and basis for the auxiliary recognition of mental illness.

\subsection{Analysis of EEG and Physiological and Biochemical Information of Mental Diseases Based on Artificial Intelligence}

Studies have shown that EEG signals have high time resolution and high sensitivity, and can be used as a reliable indicator of depression identification. Combine EEG signals with traditional feature extraction algorithms and machine learning, and then implement a supervised learning strategy to distinguish depression patients from normal subjects, which will provide future design and application of portable depression aid recognition systems based on EEG signals It's possible [8]. Jiang [9] and others collected static MEG data with closed eyes, combined with MEG tracing data and machine learning technology to establish a machine learning model to automatically .

distinguish between $\mathrm{BD}$, unipolar depression and healthy subjects. In this way, individualized and objective predictions of the types of mood disorders are carried out, which in turn has an impact on the accuracy of diagnosis and treatment decisions of patients with mood disorders.

\subsection{Application of Artificial Intelligence in Mental Disease Early Warning and Intervention}

The prevalence of mental illness and suicide rate often bring adverse effects and heavy burdens on the family and society. Early and timely identification of negative emotions is the key to preventing adverse consequences. SilverLogicLabs, an artificial intelligence start-up company in Seattle, USA, has developed a dynamic expression recognition system that can monitor and judge the emotional state of people in real time, and can be used to detect emotional characteristics of patients.

Passos [10] and others used clinical variables and demographic variables related to suicide attempts of patients with mood disorders, and used "training" machine learning algorithms to implement and evaluate three machine learning algorithms: minimum absolute contraction and selection operator SVM and correlation vector The results show that the three algorithms can distinguish suicide attempters from non-suicides, and their accuracy is between $65 \%$ and $72 \%(\mathrm{P}<0.05)$. In particular, the accuracy of RVM algorithm prediction reached $72 \%$ (sensitivity and specificity were $72.1 \%$ and $71.3 \%$, respectively) and the area under the curve of the receiver operating characteristic curve was $0.77(\mathrm{P}<0.0001)$. It is suggested that artificial intelligence algorithms can be used to assess the suicide risk of patients with mood disorders by combining demographic and clinical variables, so as to achieve the purpose of suicide warning.

In 2017, Facebook company developed a psychological robot Woebot, through a structured dialogue system, based on deep learning natural language understanding, from the user's language to detect and evaluate their negative emotions, and even serious mental illness tendencies. After understanding and evaluating the user's mental health, it is recommended to consult professional psychologists and physicians to achieve early identification and intervention of mental illness.

\subsection{The advantages and disadvantages of artificial intelligence in assisting the treatment of mental illness}

We use online and offline questionnaire survey methods to collect public opinions. The method is to ask questions in writing to understand the opinions of different age groups. genders,occupations, and the public on artificial intelligence-assisted psychotherapy, so as to draw artificial intelligence-assisted mental illness treatment Market demand.

The survey results show that the public believes that the advantages of artificial intelligence-assisted mental illness treatment in the medical field are analyzed (see Figure 1 for details), and they believe that the advantage of more accurate diagnosis accounts for the highest proportion, which is $74.12 \%$, followed by improving the efficiency of diagnosis. , Accounting for $42.63 \%$, narrowing the gap between hospitals and sharing medical resources, accounting for $41.83 \%$ and $32.6 \%$ respectively. At present, the public pays more attention to the accuracy of diagnosis and the efficiency of treatment for the treatment of mental illness.

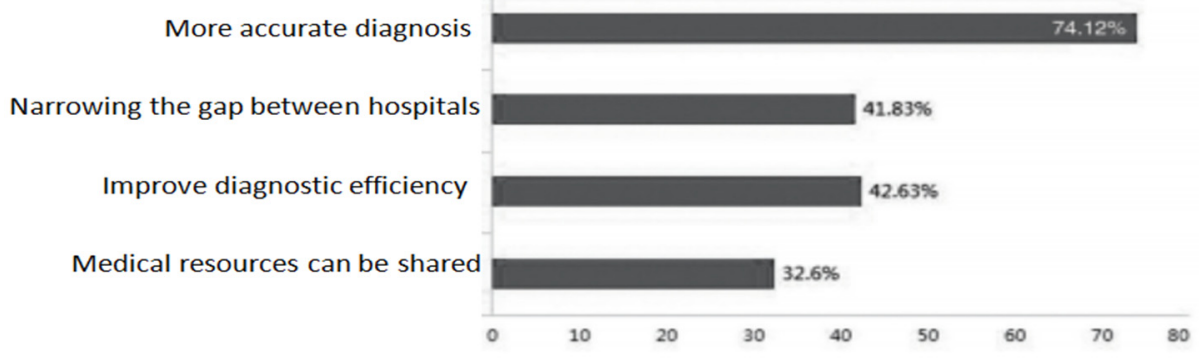

Figure 1. the advantages of artificial intelligence assisted mental illness treatment 
In recent years, with the continuous improvement of my country's medical standards, the misdiagnosis rate has shown a downward trend. However, the development of artificial intelligence-assisted mental illness treatment technology is not perfect, and there are still many drawbacks (see Figure 2 for details). "Patients' private information is leaked", "treatment costs are expensive", and "employment income of workers with mental illness is affected" accounted for $40.52 \%, 24.27 \%$ and $14.24 \%$, respectively. But the highest proportion is "intelligent technology development is not mature enough, afraid of misdiagnosis". It can be seen that in order to achieve rapid development of this kind of treatment, all aspects need to be continuously improved.

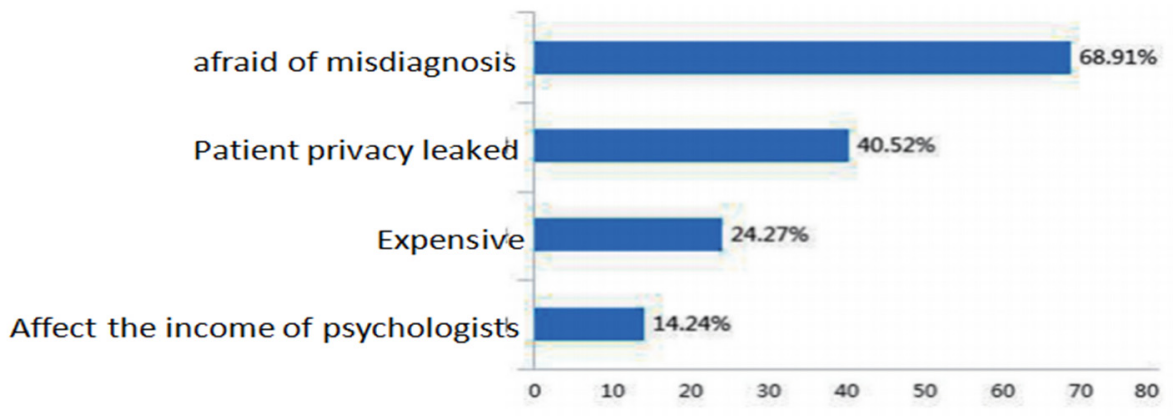

Figure 2. disadvantages of artificial intelligence-assisted mental illness treatment

\section{The application of artificial intelligence to the psychological level of the elderly}

In recent years, the research topics and projects of intelligent elderly care have continuously entered the public's field of vision. At the same time, people are increasingly calling for the development of artificial intelligence robots for elderly care services to make up for the shortage of elderly personnel and resources. The development of artificial intelligence robots for elderly care services can effectively alleviate the pressure on the family, government and society for elderly care. For example, artificial intelligence robots instead of artificially taking care of the elderly can effectively alleviate the burden of serious shortage of elderly care personnel. Even the use of effective resources such as the Internet, the Internet of Things, and big data to alleviate the problem of the low number and level of equipment in elderly care institutions is conducive to improving the quality of life of the elderly in their later years, which is of great significance to the stable operation of the entire society. However, the current researches on artificial intelligence robots for elderly care services are mostly focused on how to improve the quality of physical services for the elderly, and there is still relatively insufficient attention to the mental health of the elderly. According to Maslow's needs theory, the emotional comfort and spiritual needs of the elderly cannot be ignored. Solving the problem of the spiritual emptiness of the elderly plays an extremely important role in their later life.

Artificial intelligence robots control brain components and systems, introduce relatively mature intelligent sensing technology, and support the entire system based on GIS, GPS, TTS, mobile APP (client software) and other technologies. The artificial intelligence robot completes the integrated control of the brain system such as voice interaction, walking, image recognition, navigation and positioning, and realizes the use of big data analysis to ensure the complete operation of the system. Pay attention to the mental state of the elderly in real time, provide timely service functions such as family companionship, emotional judgment, game amusement, cultural services, and smart chat to meet the mental and social needs of the elderly, thereby reducing the anxiety and loneliness of the elderly, etc. situation. At the same time, it can also transfer the mental changes of the elderly to the family members of the elderly through the one-key transfer function.

We can introduce Bayesian rules to further analyze the probability of artificial intelligence emotion recognition. First, we denote the third-person evaluation process as $\mathrm{P}(\mathrm{e}$ o), which is the set of probability distributions of sentiment (e) for a given result (o). We ignore the beliefs and desires of the actors in the evaluation process (this article will discuss the beliefs and desires of the actors in the "reverse evaluation" below).

According to Bayes' rule, we can first write the posterior probability $\mathrm{P}(\mathrm{o} \mid \mathrm{e})$ of the emotion, that is, the probability of the result (o) of a given emotion (e) is the formula (1): $\mathrm{P}(\mathrm{o} \mid \mathrm{e}) \propto \mathrm{P}(\mathrm{e} \mid \mathrm{o}) \mathrm{P}(\mathrm{o})$

When the observer faces different clues, he needs a more precise theory to integrate multiple clues. How the observer should integrate these different clues information requires an accurate and optimized formula to analyze. The integration of emotional cues involves a high-level reasoning. Assuming that given an observation (o) and emotional expression $(\mathrm{x}), \mathrm{P}(\mathrm{e} \mid \mathrm{o}, \mathrm{x})$, the probability of potential emotion (e) can be expressed by the following formula (2): $\mathrm{P}(\mathrm{e} \mid \mathrm{o}, \mathrm{x}) \propto \mathrm{P}(\mathrm{o}) \mathrm{P}(\mathrm{e} \mid \mathrm{o}) \mathrm{P}(\mathrm{e} \mid \mathrm{x}) \mathrm{P}(\mathrm{e})$

It should be noted that this probabilistic inference also integrates the emotional probability $\mathrm{P}(\mathrm{e} \mid \mathrm{o})$ of a given result and the emotional probability $\mathrm{P}(\mathrm{e} \mid \mathrm{x})$ of a given emotional expression. The model does not assume the priority of any kind of cues, such as which facial expression is more prioritized, but measures these cues based on the reliability of the actor's potential emotions.

The impact of social robots on depression of healthy elderly people is reported on the depression scores of people with dementia. The statistical heterogeneity is $(\mathrm{P}=$ $0.23, \mathrm{I} 2=30 \%$ ). Therefore, a fixed-effect model is used. The results show that social robots are Compared with the 
control group, the senile dementia population can improve the score of depression, the difference is statistically significant $(\mathrm{SMD}=-0.49,95 \% \mathrm{CI}(-0.80 \sim-0.18), \mathrm{Z}=3$. 08, $\mathrm{P}=0.002]$.

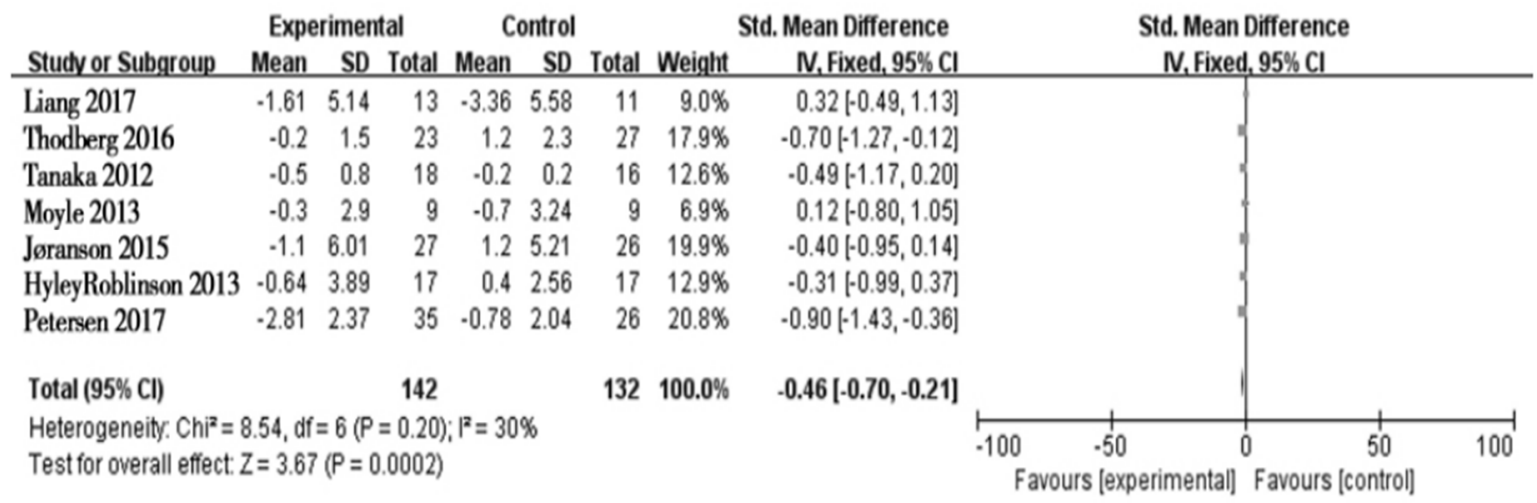

Figure 3. Meta-analysis forest diagram of social robots in the treatment of senile depression

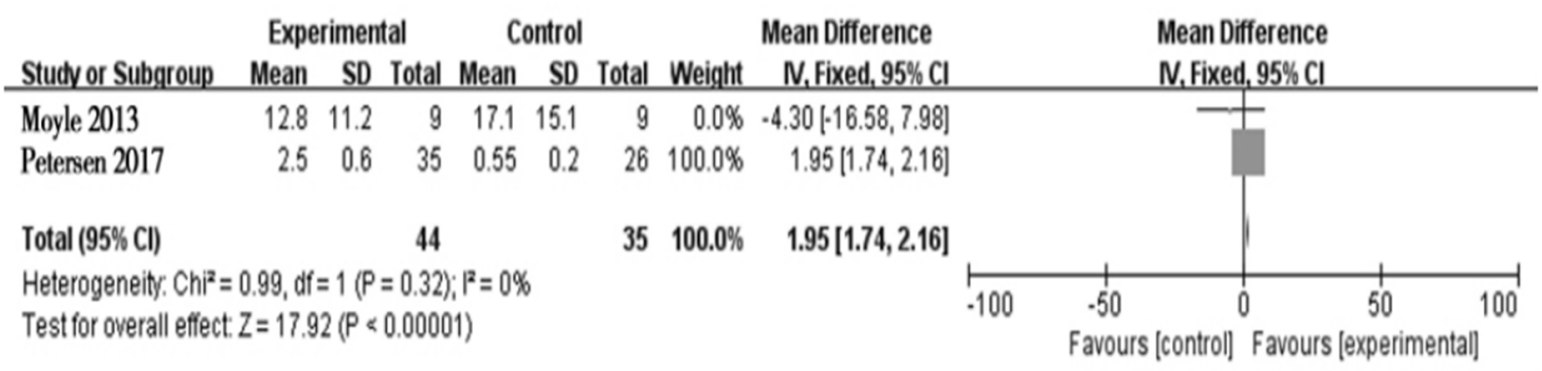

Figure 4. Meta-analysis forest diagram of social robots for the treatment of elderly anxiety

The impact of social robots on elderly anxiety, a total of 79 elderly people, the statistical heterogeneity is $(\mathrm{P}=$ $0.32, \mathrm{I} 2=0 \%$ ), the fixed-effects model Meta analysis results show that the social robot group is significantly better than conventional nursing in the treatment of elderly anxiety.In the management group, the difference was statistically significant $[\mathrm{MD}=1.95,95 \% \mathrm{CI}(1.74 \sim 2.16)$, $\mathrm{Z}=17.92, \mathrm{P}<0.00001]$

\section{Conclusion}

Doctors can use artificial intelligence technology to further integrate early identification, early warning and treatment of mental illness, so that machines or smart devices can replace doctors and nurses to independently complete some simple and repetitive tasks, which can save medical resources, improve work efficiency, and relieve psychiatric professional resources services to a certain extent.The scarcity status is also expected to realize personalized remote intervention of mental illness. Moreover, it is possible to use artificial intelligence technology to collect and analyze various data such as daily activities, emotional changes, and physical health of the elderly through services such as Internet + and smart sensor equipment, and pay attention to their mental health in time, and provide emotional support and emotion comfort. The research and development of artificial intelligence robots that improve the quality of life and happiness of the elderly is in line with the trend of the times and is also an important development direction in the artificial intelligence era. It is of great significance to solve the current increasingly severe aging problem. Its development depends on both the progress of science and technology and the research in psychology. It is also inseparable from the researcher's "humanism" and the spiritual needs of the elderly.

\section{References}

1. [English] Edited by Margaret A. Boden. Philosophy of Artificial Intelligence [M]. Liu Xirui, translated by Wang Hanqi. Shanghai Translation Publishing House, 2005.

2. Cai Zixing, Xu Guangku. Artificial Intelligence and Its Applications (Fourth Edition) [M]. Tsinghua University Press, 2010.

3. TopolEJ.Highperformancemedicine:theconvergenceofhumanandart ificialintelligence[J].NatMed,2019,25(1):4456.DOI:10.1038/s41591-018-0300-7.

4. BrinkerTJ,HeklerA,HauschildA, etal.Comparingartifi cialintelligencealgorithmsto157Germandermatologis ts:themelanomaclassificationbenchmark[J].EurJCanc er,2019,111(6):30-

37.DOI:10.1016/j.ejca.2018.12.016. 
5. WangY,KungLA,ByrdTA.Bigdataanalytics:understan dingitscapabilitiesandpotentialbenefitsforhealthcareo rganizations[J].TechnolForecastSocChange, 2016,126 (3):3-13.DOI:10.1016/j.techfore.2015.12.019.

6. CannizzaroM,HarelB,ReillyN,etal.Voiceacousticalm easurementoftheseverityofmajordepression.Brainand Cognition,2004,56(1):30-35

7. GillespieS,LauresGoreJ,MooreE,etal.Identificationof AffectiveStateChangeinAdultsWithAphasiaUsingSp eechAcoustics.JSpeechLangHearRes,2018,61(12):29 06-2916

8. LiX,ZhangX,ZhuJ,etal.Depressionrecognitionusingm achinelearningmethodswithdifferentfeaturegeneratio nstrategies.ArtificialIntelligenceinMedicine,2019,99: 101696

9. JiangH,DaiZ,LuQ,etal.Magnetoencephalographyresti ngstatespectralfingerprintsdistinguishbipolardepressi onandunipolardepression.BipolarDisord,2019,doi:10. 1111/bdi.12871

10. PassosIC,MwangiB,CaoB,etal.Identifyingaclinicalsi gnatureofsuicidalityamongpatientswithmooddisorder s:Apilotstudyusingamachinelearningapproach.JAffec tDisord,2016,193:109-116G. Eason, B. Noble, and I. N. Sneddon, "On certain integrals of LipschitzHankel type involving products of Bessel functions," Phil. Trans. Roy. Soc. London, vol. A247, pp. 529551, April 1955. (references) 